\title{
Diseño de Recursos Educativos Abiertos con GeoGebra para el estudio de la Generalización de Patrones
}

\author{
Ma. Brisa Vázquez-Macuitl ${ }^{1}$, Francisco J. Anaya Puebla ${ }^{2}$, Sergio Rubio-Pizzorno ${ }^{3}$ \\ ${ }^{1}$ Centro Escolar Licenciado Miguel Alemán (Celma) - Benemérita Universidad \\ Autónoma de Puebla (Buap) - México. \\ ${ }^{2}$ Benemérita Universidad Autónoma de Puebla (Buap), México. \\ ${ }^{3}$ Instituto GeoGebra Internacional, México/Chile \\ brisavazquezmacuitl@gmail.com, paco_anaya@hotmail.com, \\ sergio.rubio@cinvestav $\cdot \mathrm{mx}$
}

Abstract. This article reports the design phase of an educational resource for the study of the generalization of patterns, within the framework of a Design-Based Research. Specifically, the methodological design called the Negotiation Process is put into operation, in which consensus is sought between research results, teaching experience and the recognition of Hybrid Educational Ecosystems. As a result of this process, an open educational resource has been elaborated, articulated in a GeoGebra Book, for the study of patterns in different registers, such as the graphic register, natural language, tabular and algebraic.

Resumen. En este artículo se reporta la fase de diseño de un recurso educativo para el estudio de la generalización de patrones, en el marco de una Investigación Basada en el Diseño. Específicamente se pone en funcionamiento el diseño metodológico llamado Proceso de Negociación, el en cual se busca el consenso entre los resultados de investigación, la experiencia docente y el reconocimiento de los Ecosistemas Educativos Híbridos. Como resultado de este proceso se ha elaborado un recurso educativo abierto, articulado en un Libro GeoGebra, para el estudio de patrones en diferentes registros, tales como, el registro gráfico, lenguaje natural, tabular y algebraico. 
VIII Congresso Brasileiro de Informática na Educação (CBIE 2019)

Anais dos Workshops do VIII Congresso Brasileiro de Informática na Educação (WCBIE 2019)

\section{Problemática}

En el Currículo de Educación Media Superior, campo disciplinar de Matemáticas de Bachillerato General y Bachillerato Tecnológico de la Secretaría de Educación Pública en México, se menciona en repetidas ocasiones la importancia de los patrones, específicamente en las asignaturas de Matemáticas I - Del pensamiento aritmético al pensamiento algebraico y Matemáticas IV - Pensamiento y lenguaje variacional.

En estas asignaturas se consideran los siguientes aprendizajes esperados: i) expresar de forma coloquial y escrita fenómenos de su vida cotidiana con base en prácticas como: simplificar, sintetizar, expresar, verbalizar, relacionar magnitudes, generalizar patrones, representar mediante símbolos, comunicar ideas, entre otras, y ii) construir y analizar sucesiones numéricas y reconocer patrones de crecimiento y decrecimiento, respectivamente [Secretaría de Educación Pública de México, 2017]. De lo anterior se desprende la necesidad de contar con recursos educativos que ayude al estudiante en la transición del pensamiento aritmético al algebraico.

Desde la aparición del software libre GeoGebra, éste ha sido un aporte a la innovación de las estrategias de enseñanza en matemáticas, puesto que cada vez más docentes integran a su práctica el usos del ambiente de geometría dinámica de GeoGebra. Este ambiente permite explorar, probar, generar datos, construir y generar nuevas preguntas sobre las matemáticas [Rubio-Pizzorno,2018], con lo cual los alumnos tienen la oportunidad de interactuar con los objetos matemáticos de una manera dinámica.

Como respuesta a esta problemática, en el presente trabajo se presenta un diseño educativo para ayudar a los estudiantes en la transición del pensamiento aritmético al algebraico, aprovechando el potencial del software GeoGebra y sus Herramientas de Autor para la elaboración de recursos educativos abiertos.

\section{Referentes teóricos}

El diseño educativo que reportamos en el presente trabajo está elaborado con base en dos fundamentos teóricos, a saber, el trabajo de López-Acosta (2016) sobre la generalización de patrones usando estrategias del pensamiento y lenguaje variacional, y la propuesta de Ursini, Escareño, Montes y Trigueros (2005) sobre los diferentes usos de la variable. A continuación se mencionan los elementos que se retoman de ambos fundamentos teóricos para la elaboración del diseño educativo:

\subsection{Pensamiento y Lenguaje Variacional}

López-Acosta (2016) enmarca su investigación en la Teoría Socioepistemológica de la Matemática Educativa, la cual señala que el conocimiento matemático es construido con base en prácticas sociales. Específicamente se centra en las situaciones de variación donde hace uso de estrategias variacionales como un punto de partida para el análisis y reflexión acerca del cambio y sus efectos. 
Las estrategias de variación consisten en formas particulares de razonar y actuar ante situaciones variacionales con la finalidad de reconocer y estudiar cualitativa o cuantitativamente los cambios de las variables involucradas. Algunas de estas estrategias son: la comparación, que permite identificar la transformación que de un valor para convertirse en otro; la seriación, permite identificar patrones en el comportamiento de los cambios mediante el análisis de las relaciones entre colecciones de comparaciones; la predicción se refiere a la anticipación de un comportamiento en la situación variacional; y la estimación que es la predicción en términos globales sobre un comportamiento para determinar la tendencia de la situación variacional.

\subsection{Modelo 3UV}

En respuesta al objetivo de ayudar a la transición del pensamiento aritmético al algebraico, en este trabajo se reconoce la importancia de la variable en álgebra y que puede manifestar diferentes usos, según el tipo de contexto o situación.

Ursini et al. (2005) proponen que los usos de la variable más comunes son como incógnita, número general y relación funcional. Cada uno de estos usos contempla distintos niveles de abstracción sobre el manejo de la variable, los cuales se muestran en la Tabla 1. Estos corresponden a aspectos importantes para que los estudiantes trabajen exitosamente con problemas y ejercicios que involucran la variable en estos tres usos durante las tareas de reconocimiento de patrones.

\subsection{Ecosistemas Educativos Híbridos}

Rubio-Pizzorno (2018) destaca que los los ambientes educativos y, específicamente los de diseño, ya no son espacios discretos y aislados unos de otros, sino que se construyen de manera simbiótica aprovechando su valor epistémico [Artigue, 2002], respecto de estudiar alguna noción matemática. A este tipo de ambientes o ecosistemas educativos les denomina híbridos, puesto que se constituyen a partir de soportes materiales de diversas naturalezas, como por ejemplo ambientes físicos (lápiz y papel), digitales (ambiente de geometría dinámica de GeoGebra), de realidad aumentada, entre otros.

Con base en el reconocimiento de la naturaleza híbrida de los ecosistemas educativos, en este trabajo se propone elaborar un recurso educativo que considere la articulación de diferentes ambientes de diseño, como lápiz y papel, el ambiente de geometría dinámica de GeoGebra y las Herramientas de Autor de GeoGebra para elaborar un recurso educativo abierto (REA) de tipo colección [Willey, Bliss y McEwen, 2014], llamada Libro GeoGebra. 
VIII Congresso Brasileiro de Informática na Educação (CBIE 2019)

Anais dos Workshops do VIII Congresso Brasileiro de Informática na Educação (WCBIE 2019)

Tabla 1: Uso de la Variable

\begin{tabular}{|c|c|c|}
\hline Número General & Incógnita & Relación Funcional \\
\hline $\begin{array}{l}\text { (G1) Reconocer patrones, percibir } \\
\text { reglas y métodos en secuencias y } \\
\text { en familias de problemas. } \\
\text { (G2) Interpretar un símbolo como } \\
\text { la representación de una entidad } \\
\text { general indeterminada que puede } \\
\text { asumir cualquier valor. } \\
\text { (G3) Deducir reglas y métodos } \\
\text { generales en secuencias y familias } \\
\text { de problemas. } \\
\text { (G4) Manipular (simplificar, } \\
\text { desarrollar) la variable simbólica. } \\
\text { (G5) Simbolizar enunciados, } \\
\text { reglas o métodos generales. }\end{array}$ & 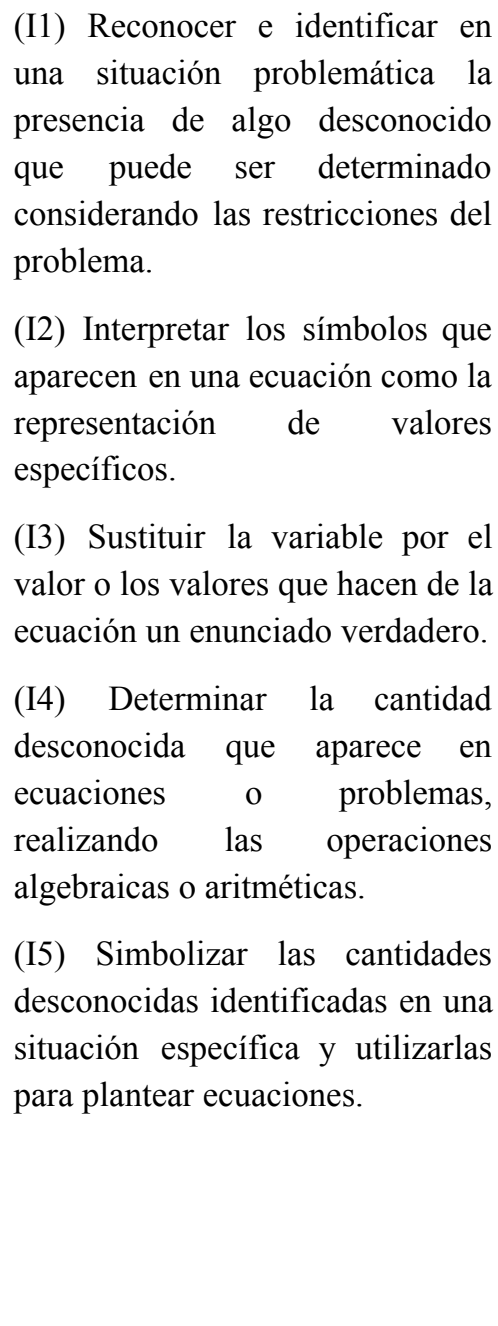 & $\begin{array}{l}\text { (F1) Reconocer la } \\
\text { correspondencia entre variables } \\
\text { relacionadas, independientemente } \\
\text { de la representación utilizada } \\
\text { (tablas, gráficas, problemas } \\
\text { verbales, expresiones analíticas). } \\
\text { (F2) Determinar los valores de la } \\
\text { variable dependiente, dados los } \\
\text { valores de la independiente. } \\
\text { (F3) Determinar los valores de la } \\
\text { variable independiente, dados los } \\
\text { valores de la dependiente. } \\
\text { (F4) Reconocer la variación } \\
\text { conjunta de las variables } \\
\text { involucradas en una relación } \\
\text { funcional, independientemente de } \\
\text { la representación utilizada (tablas, } \\
\text { gráficas, problemas verbales, } \\
\text { expresiones analíticas). } \\
\text { (F5) Determinar los intervalos de } \\
\text { variación de una de las variables, } \\
\text { dado el intervalo de variación de } \\
\text { la otra. Simbolizar una relación } \\
\text { (F6) basados en el análisis } \\
\text { funcional, basabs } \\
\text { de los datos de un problema. }\end{array}$ \\
\hline
\end{tabular}

\subsection{Principios para el diseño del REA}

De cada uno de los referentes teóricos tomamos elementos que van a servir como principios de diseño del REA, es decir, que van a orientar sus objetivos, las actividades y la articulación entre ellas.

De la investigación de López-Acosta (2016) se considera el uso de las estrategias variacionales para el estudio de elementos gráficos, las cuales son usadas en el diseño como la estructura de cada capítulo. Primero hay actividades de comparación, luego de predicción. En cada actividad se utilizan las preguntas características de las estrategias variacionales, como por ejemplo, ¿qué cambia?, ¿cómo cambia?, ¿cuánto cambia?, ¿qué no cambia?, ¿cuál es total? 
Rubio-Pizzorno [2018] reconoce la naturaleza híbrida de los ecosistemas educativos en la actualidad, a partir de lo cual consideramos el uso de diferentes ambientes de diseño para la elaboración del recurso educativo sobre patrones. Esta consideración se hace con base en la real disponibilidad de los ambientes de diseño en un aula de clases del Centro Escolar Licenciado Miguel Alemán (Puebla, México). Entre ellos encontramos lápiz y papel, manipulables como palillos, el software de matemáticas dinámicas de GeoGebra (versión de escritorio y móvil) y las Herramientas de Autor de GeoGebra (Actividades y Libro GeoGebra).

\section{Metodología}

Para la elaboración del REA con base en las ideas de la sección anterior, se siguió el diseño metodológico Proceso de Negociación de Rubio-Pizzorno (2018), el cual se enmarca en las metodologías de Investigación Basada en el Diseño [Swan, 2014].

El Proceso de Negociación consiste en un marco de búsqueda de consensos entre diferentes voces sociales que constituyen los saberes docentes [Mercado, 2002] durante la elaboración de diseños educativos. En el caso del presente trabajo tales voces sociales consideradas en el Proceso de Negociación corresponden a 1) los resultados de investigación al servicio de los docentes, 2) la experiencia docente y 3) el reconocimiento de los Ecosistemas Educativos Híbridos a través de la atención a los ambientes de diseño, tal como se muestra en la Figura 1.

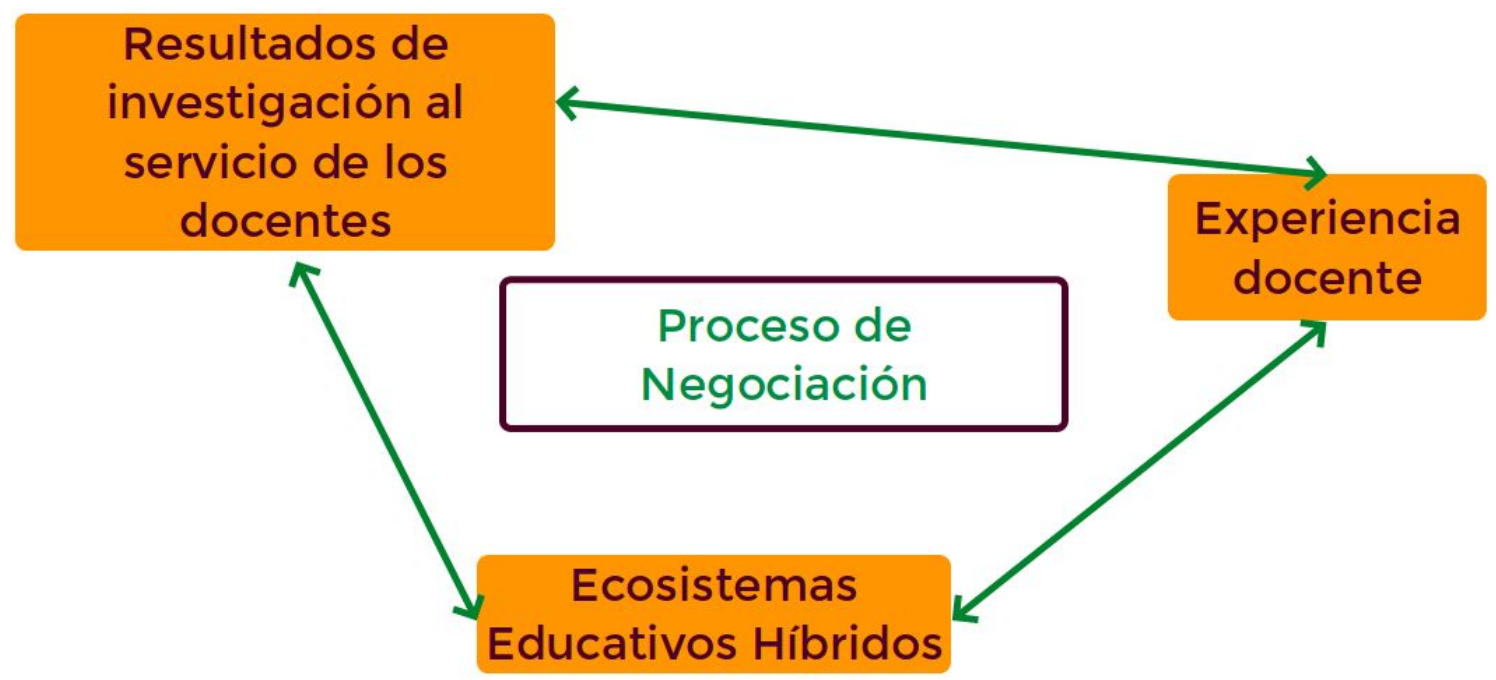

Figura 1. Esquema del Proceso de Negociación para la elaboración de diseños didácticos. Adaptado de Rubio-Pizzorno (2018, p. 148).

Este proceso de negociación fue llevado a cabo en el diplomado Diseño de Estrategias y Recursos Educativos en ambientes digitales libres dictado el primer semestre de 2019 en el Instituto Nacional de Astrofísica, Óptica y Electrónica (Puebla, México), impartido por el profesor Sergio Rubio-Pizzorno. El diplomado estaba conformado por varias profesoras y profesores de diferentes niveles educativos (primaria, secundaria, bachillerato y superior), quienes participaron siempre del proceso de negociación para 
VIII Congresso Brasileiro de Informática na Educação (CBIE 2019)

Anais dos Workshops do VIII Congresso Brasileiro de Informática na Educação (WCBIE 2019)

definir todos los aspectos del REA a elaborar, configurando así el polo de experiencia docente del proceso de negociación.

Para configurar el polo de resultados de investigación se revisaron las actividades propuestas por López-Acosta (2016) para analizar cuáles se utilizarían para el diseño referentes al análisis geométrico, por otra parte se decidió incorporar al modelo $3 \mathrm{UV}$ ya que nos permitió identificar y clasificar el uso de la variable en distintos contextos.

El polo de atención al ambiente del diseño se configuró a partir de plantearse que en el REA se articularan actividades construidas en ambientes físicos (lápiz y papel) y digital (ambiente de geometría dinámica de GeoGebra y Libro GeoGebra).

Con los polos del proceso de negociación ya constituido, la elaboración del REA siguió el siguiente proceso: búsqueda de fundamentos teóricos de la Matemática Educativa para orientar el diseño de las actividades (ver sección 2. Referentes teóricos), planificación del REA, creación de applets, diseño del material didáctico, y finalmente la estructuración de estas en un Libro GeoGebra.

Luego de hacer la búsqueda de fundamentos teóricos, se pasó a realizar una planificación del REA que permitiera tener claridad de los objetivos a abordar y sus diferentes partes. Para ello se utilizó como estructura de la planificación la Trayectoria Hipotética de Aprendizaje (THA) [Simon, 1995], la cual consiste en determinar un objetivo del REA, las tareas asociadas a tal objetivo y el proceso hipotético de aprendizaje ligado a las tareas.

En Vázquez-Macuitl, Anaya y Rubio-Pizzorno (2019) puede acceder a la versión completa de la planificación. Como objetivo del REA se consensuó el siguiente: desarrollar procesos cognitivos que les permitan a los estudiantes la transición del pensamiento aritmético al algebraico a partir de recursos híbridos de aprendizaje que presenten patrones o reglas de secuencias de objetos que pueden ser numéricos o geométricos.

A partir de este objetivo y las tareas que se desprenden de él, se diseñaron actividades geométricas para establecer las relaciones algebraicas vistas no sólo como expresiones abstractas; la manipulación de los elementos en los applets, las preguntas generadoras y una serie de acciones por las cuales el estudiante transita entre los ambientes físicos y digitales, permite reconocer y establecer las relaciones entre los objetos, guiando al alumno por las actividades acorde a las estrategias del pensamiento variacional iniciando por la comparación hasta determinar la tendencia situacional. De manera general, las actividades están diseñadas con la estructura que muestra la Figura 2 , cuyos aspectos son retomados de los referentes teóricos sobre los usos de la variable y las estrategias variacionales. 


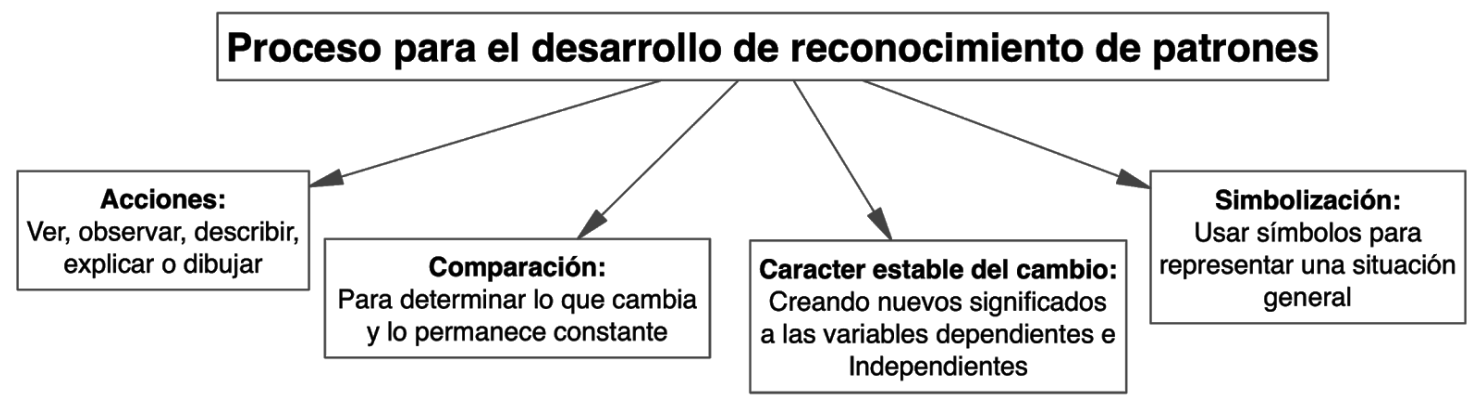

Figura. 2. Proceso para el desarrollo de reconocimiento de patrones

\section{Descripción de las actividades del REA}

A continuación se presentan actividades del Libro GeoGebra Comprensión de patrones (Anaya, 2019), específicamente del Capítulo I: Secuencias Lineales, cuyo objetivo es que los estudiantes identifiquen patrones lineales con elementos constantes.

Actividad 1.1 - Secuencia lineal sin elementos constantes (Figura 3), en esta actividad se presenta un applet en el cual el estudiante puede observar el comportamiento del patrón al manipular el deslizador $n$, o al activar cualquiera de los dos botones los cuales incrementan o decrementan en una unidad el valor de la variable n. La tareas a realizar por parte de estudiante son: 1) explicar en lenguaje natural el comportamiento del patrón, 2) dibujar en su libreta el caso 12, 3) completar una tabla con datos faltantes, 4) explicar el comportamiento del patrón a otra persona, 4) proponer una expresión para calcular la cantidad de rombos para cualquier $n$.

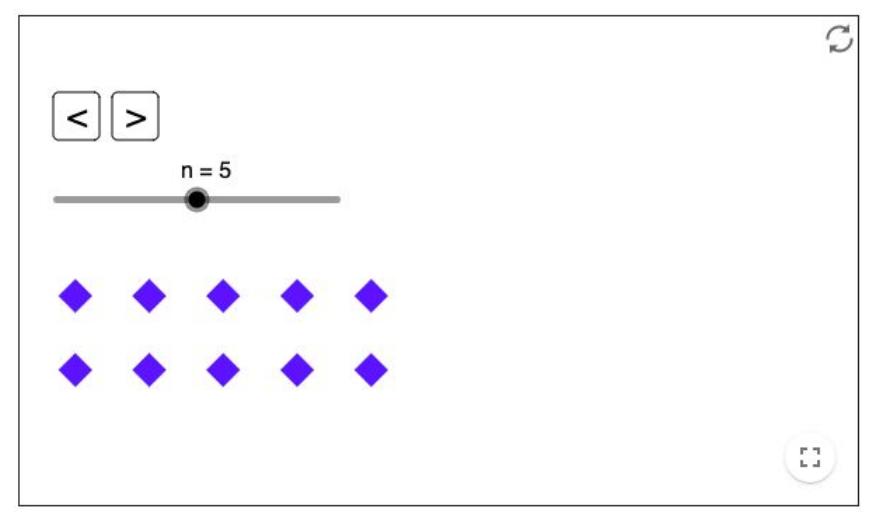

Figura 3. Secuencia lineal sin elementos constantes

Actividad 1.3 - Secuencia lineal con elementos constantes (Figura 4), en esta actividad se presenta un applet en el cual el estudiante puede observar el comportamiento del patrón al manipular el deslizador $n$, o al presionar cualquiera de los dos botones para incrementar o decrementar en una unidad el valor de la variable $n$. La tareas a realizar son idénticas a la tarea 1.1. 


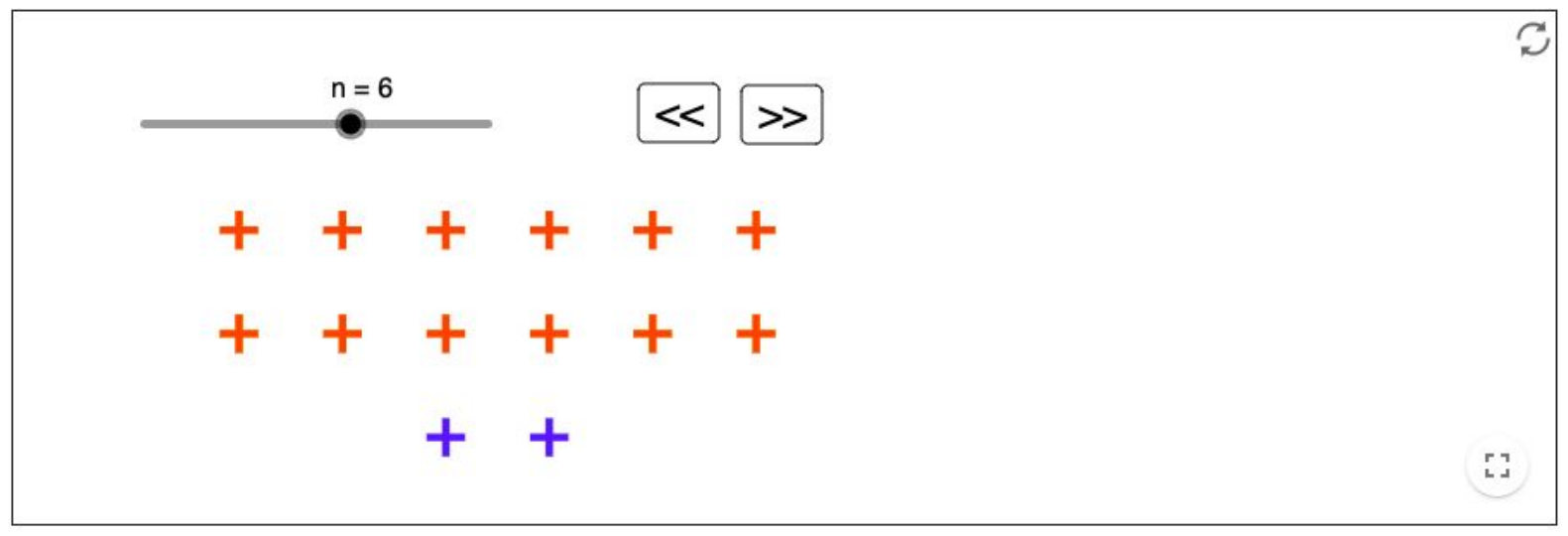

Figura 4. Secuencia lineal con elementos constantes

En la actividad 1.3 se solicita al estudiante realizar las siguientes tareas: 1) identificar similitudes y diferencias en el comportamiento de los patrones analizados en las dos tareas anteriores, 2) responder a la pregunta ¿qué se tiene que hacer para determinar el número de rombos o cruces de una figura cualquiera de la secuencia?

Por último en la actividad 1.4 se proponen una serie de expresiones algebraicas que podrían explicar el comportamiento de los dos patrones explorados y se plantean preguntas al estudiante como por ejemplo dada la expresión $k=2 n+2: 1)$ ¿Qué representa la letra $n$ en las expresión anterior?,2) ¿Qué representa la letra k en las expresión anterior?

Los capítulos siguiente permiten al estudiante explorar algunos otros patrones geométricos utilizando una estructura muy similar a la presentada en el Capítulo I.

\section{Conclusiones y prospectivas}

A partir de los referentes teóricos y la manera de elaborar el REA durante el Proceso de Negociación, se espera que responda a las necesidades educativas de los estudiantes en condiciones reales de clase. Para ello, será necesario realizar la implementación de este recurso, la que permita llevar a cabo un proceso de tomar de datos cuidada y un posterior análisis. Con los resultados de tal análisis se espera obtener mayor información para poder realizar un rediseño del recurso, de tal manera de ir ajustando cada vez más cada una de las variables involucradas en el recurso y puestas a prueba en su implementación con estudiantes. 
VIII Congresso Brasileiro de Informática na Educação (CBIE 2019)

Anais dos Workshops do VIII Congresso Brasileiro de Informática na Educação (WCBIE 2019)

\section{Referencias}

Anaya, F. (2019). Comprensión de patrones [Libro GeoGebra]. Rescatado de https://www.geogebra.org/m/tnk9bve4

Artigue, M. (2002). Learning mathematics in a CAS environment: The genesis of a reflection about instrumentation and the dialectics between technical and conceptual work. International Journal of Computers for Mathematical Learning, 7(3), pp. 245-274. ISSN 13823892. doi: 10.1023/A:1022103903080.

López-Acosta, L. (2016). Generalización de patrones. Una trayectoria hipotética de Aprendizaje basada en el Pensamiento y Lenguaje Variacional. Tesis de Maestría no publicada. México: Cinvestav.

Mercado, R. (2002). Los saberes docentes como construcción social. La enseñanza centrada en los niños. Fondo de Cultura Económica, D.F., México.

Rubio-Pizzorno, S. (2018). Integración digital a la práctica del docente de geometría. Tesis de Maestría no publicada. Ciudad de México, México: Centro de Investigaciones $\mathrm{y}$ de Estudios Avanzados (Cinvestav). doi: 10.13140/RG.2.2.15488.94728/1.

Secretaría de Educación Pública de México (2017). Planes de Estudio de Referencia del Marco Curricular Común de la Educación Media Superior. Recuperado el 26 de septiembre de 2019, de: https://www.gob.mx/cms/uploads/attachment/file/241519/planes-estudio-sems.pdf.

Simon, M. (1995). Reconstructing mathematics pedagogy from a constructivist perspective. Journal for Research in Mathematics Education, 26(2), pp. 114-145. ISSN 0021-8251. doi: 10.2307/749205.

Vázquez-Macuitl, M., Anaya, F. y Rubio-Pizzorno, S. (2019). Trayectoria Hipotética de Aprendizaje: Identificación de patrones geométricos como introducción al desarrollo del lenguaje algebraico. Recuperado de https://drive.google.com/file/d/1LczF0knhNJiy4j6SgHOSbxNzrOfE0z5S/view?usp= sharing

Ursini, S., Escareño, F., Montes, D. y Trigueros, M. (2005). Enseñanza del álgebra elemental. Una propuesta alternativa. México: Editorial Trillas, ISBN 968-24-6752-7.

Swan, M. (2014). Design Research in Mathematics Education. En: Stephen Lerman (Ed.), Encyclopedia of Mathematics Education, pp. 148-152. Springer, Dordrecht. DOI: 10.1007/978-94-007-4978-8_180.

Wiley, D., Bliss, T.L. y McEwen, M. (2014). Open Educational Resources: A Review of the Literature. En (Eds.) J. Michael Spector, M. David Merrill Jan Elen, M.J. Bishop. Handbook of Research on Educational Communications and Technology (pp. 781 - 789). New York: Springer Science+Business Media. ISBN: 978-1-4614-3185-5. DOI: 10.1007/978-1-4614-3185-5 